\title{
The Quantum Hall Transition in Real Space: From Localized to Extended States
}

\author{
K. Hashimoto,,${ }^{1,2,3, *}$ C. Sohrmann, ${ }^{4}$ J. Wiebe, ${ }^{1}$ T. Inaoka, ${ }^{5}$ F. Meier, ${ }^{1, \oplus}$ \\ Y. Hirayama, ${ }^{2,3}$ R. A. Römer, ${ }^{4}$ R. Wiesendanger, ${ }^{1}$ and M. Morgenstern ${ }^{6,7}$ \\ ${ }^{1}$ Institute of Applied Physics, Hamburg University, Jungiusstraße 11, D-20355 Hamburg, Germany \\ ${ }^{2}$ Department of Physics, Tohoku University, Sendai 980-8578, Japan \\ ${ }^{3}$ ERATO Nuclear Spin Electronics Project, Sendai 980-8578, Japan \\ ${ }^{4}$ Department of Physics and Center for Scientific Computing, \\ University of Warwick, Gibbet Hill Road, Coventry CV4 7AL, UK \\ ${ }^{5}$ Department of Physics and Earth Sciences, University of the Ryukyus, \\ 1 Senbaru, Nishihara, Okinawa 903-0213, Japan \\ ${ }^{6}$ II. Institute of Physics B, RWTH Aachen University, Aachen, D-52056, Germany \\ ${ }^{7}$ JARA-Fundamentals of Future Information Technology
}

(Dated: October 25, 2018)

\begin{abstract}
Using scanning tunneling spectroscopy in ultra-high vacuum at low temperature $(T=0.3 \mathrm{~K})$ and high magnetic fields $(B \leq 12 \mathrm{~T})$, we directly probe electronic wave functions across an integer quantum Hall transition. In accordance with theoretical predictions, we observe the evolution from localized drift states in the insulating phases to branched extended drift states at the quantum critical point. The observed microscopic behavior close to the extended state indicates points of localized quantum tunneling, which are considered to be decisive for a quantitative description of the transition.

PACS numbers: 73.43.Nq, 73.20.At, 73.43.Cd
\end{abstract}

Two-dimensional electron systems (2DES) are paradigms for quantum phase transitions [1]. For example, they exhibit metal-insulator, integer quantum Hall (IQH), and superconductor-insulator transitions [2], all described fairly successfully by a universal percolationtype model [3]. Some macroscopic aspects of this percolation have been verified, e.g. by transport experiments revealing critical exponents for the divergence of the localization length [4]. However, the most fundamental aspect, i.e. how wave functions change at the transition, has not been probed experimentally. Here, we directly observe the wave functions at the IQH transition taking advantage of its tunability by a magnetic $(B)$ field.

The microscopic description of IQH transitions is theoretically well established [5, 6, 7]. The 2DES in $B$ fields exhibits discrete kinetic energies which are called Landau levels (LL). The corresponding states are subject to random potential disorder, which has primarily a semiclassical effect [5]: the electrons perform a fast cyclotron rotation within the electrostatic disorder which leads to additional drift motion along the equipotential lines [8]. Quantum mechanically, so-called drift states meander along equipotential lines with a width of about the cyclotron radius $r_{c}[\underline{6}]$. If the potential energy of the state is low (high), the drift states are closed trajectories around potential minima (maxima), i.e. they are localized and represent insulating electron phases. In the center of a LL, the adjacent trajectories merge at the saddle points of the potential leading to an extended state traversing the whole sample. It is known that this state is the quantum critical state of the IQH transition and responsible for the finite longitudinal resistance between quantized values of the Hall conductance [5, $\underline{6}, \underline{7}, \underline{9}]$.
Several experiments have addressed microscopic aspects of QH transitions [10, 11]. However, none of these techniques revealed sufficient lateral resolution to image the drift states. Only scanning tunneling spectroscopy (STS) using the adsorbate-induced 2DES at n-InAs(110) surfaces found localized drift states within a LL tail [12]. This paves the path to observe the decisive transition from localized to extended states directly. Localized states in $B$-field have also been observed on graphite, which, however, does not exhibit a pure 2DES [13].

Here, we probe the local density of states (LDOS) of the adsorbate-induced 2DES on n-InSb(110) by STS. The 2DES is prepared by depositing 0.01 monolayer of Cs atoms on cleaved $\mathrm{n}-\mathrm{InSb}(110)$ at $T=30 \mathrm{~K}$ and $p \sim 10^{-10}$ mbar 14, 15. STS is performed in-situ at $T=0.3 \mathrm{~K}$ and $B \leq 12 \mathrm{~T}[16]$ using a W-tip carefully selected by trial and error to exhibit negligible tip-induced band-bending 14, 17]. The tip is stabilized at current $I_{\text {stab }}$ and voltage $V_{\text {stab }}$, prior to measuring $\mathrm{d} I / \mathrm{d} V$ as a function of sample voltage $V_{s}$ directly by lock-in technique with modulation voltage $V_{\text {mod }}$.

First we sketch the general properties of the 2DES. Figure 1(a) shows a color scale plot of the local differential conductivity $\mathrm{d} I / \mathrm{d} V\left(V_{s}\right)$ of the 2DES measured along a straight line at $B=0 \mathrm{~T}$ and the corresponding spatially averaged $\mathrm{d} I / \mathrm{d} V$ curve. They, respectively, represent the energy dependence of the LDOS (energy-position plot) and of the macroscopically averaged LDOS, i.e. the DOS [18]. The LDOS of the 2DES shows two apparent boundaries coinciding with two step-like features in the DOS at $V_{s}=-115$ and $-47 \mathrm{meV}$, which represent the first $\left(E_{1}\right)$ and second $\left(E_{2}\right)$ subband edges as indicated in Fig. 1 (a). The subband energies are excellently reproduced by 
a self consistent calculation [Fig. 1(b)] [14, 19]. The irregularity of the onset at $E_{1}$ is a signature of the potential disorder 18]. Figure 1 (c) shows a set of $\mathrm{d} I / \mathrm{d} V$ curves measured at the same position at different $B$. Starting at $B=6 \mathrm{~T}$, the $\mathrm{d} I / \mathrm{d} V$ curves exhibit distinct LLs with a pronounced twofold spin splitting. The LLs are separated by regions of $\mathrm{d} I / \mathrm{d} V \approx 0$ evidencing complete quantization. Indeed, spin-resolved IQH plateaus up to filling factor six were recently observed by magnetotransport on an adsorbate-induced 2DES on $\operatorname{InSb}(110)$ [20]. Repeating the measurement using smaller $B$-field steps highlights the continuous evolution of the spin-split LLs, i.e. the LL fan diagram in Fig. 1(d). The green (red) dashed lines mark the four (two) spin-down LLs of the 1st (2nd) subband. The accompanying spin-up LLs are visible at higher energies as marked by blue arrows for the lowest LL. Partly, LLs of different subbands cross without anticrossing indicating orthogonality and, thus, negligible interaction between the subbands. From the peak distances, we deduce the effective mass $m^{*}$ and the absolute value of the $g$-factor $|g|$ via the separation of LLs $\hbar|e| B / m^{*}$ ( $\hbar$ : Dirac's constant, $e$ : electron charge) and of spin levels $|g| \mu_{B} B$ ( $\mu_{B}$ : Bohr magneton). For the lowest peaks at $B=6 \mathrm{~T}$, we find $m^{*} / m_{e}=0.019 \pm 0.001$ $\left(m_{e}\right.$ : free-electron mass) and $|g|=39 \pm 2$. This is close to the known values at the band edge $m^{*} / m_{e}=0.014$ and $|g|=51$ with slight deviations due to non-parabolicity and energy dependent spin-orbit coupling as known for $\mathrm{InSb}$ [21, 22].

Figures 2(a)-(g) show our central result, the LDOS across an IQH transition, i.e. $\mathrm{d} I / \mathrm{d} V$ images recorded in the lowest spin-down LL of the 2DES (LLO $\downarrow$ ) at $B=12$ T. The corresponding, spatially averaged $\mathrm{d} I / \mathrm{d} V$ curve is shown in Fig. 2(h). The continuous change of the LDOS with energy is available as an EPAPS movie 14]. The LDOS in the low-energy tail of LLO $\downarrow$ is shown in Fig. 2 (a). It exhibits spatially isolated closed-loop patterns with averaged full width at half maximum $(\mathrm{FWHM}) \simeq$ $6.9 \mathrm{~nm}$ close to $r_{c}=7.4 \mathrm{~nm}$. Thus, we attribute the closed patterns to localized drift states of LL0 $\downarrow$ aligning along equipotential lines around a potential minimum. Accordingly, at slightly higher energy [Fig. 2(b)], the area encircled by the drift states increases indicating that the drift states probe a longer equipotential line at higher energy within the same valley. In contrast, the ring patterns at the high-energy tail, marked by green arrows in Fig. 2(f), (g), encircle an area decreasing in size with increasing voltage. These states are attributed to localized drift states around potential maxima. Notice that the structures in Fig. 2(a) and (b) appear nearly identically in Fig. 2(f) and (g) as marked by white arrows. The latter structures are the LL0 $\uparrow$ states localized around potential minima, which energetically overlap with the high-energy LLO $\downarrow \downarrow$ states localized around potential maxima. When the voltage is close to the LL center [Fig. 2(c), (e)], adjacent drift states coalesce and a dense network is observed
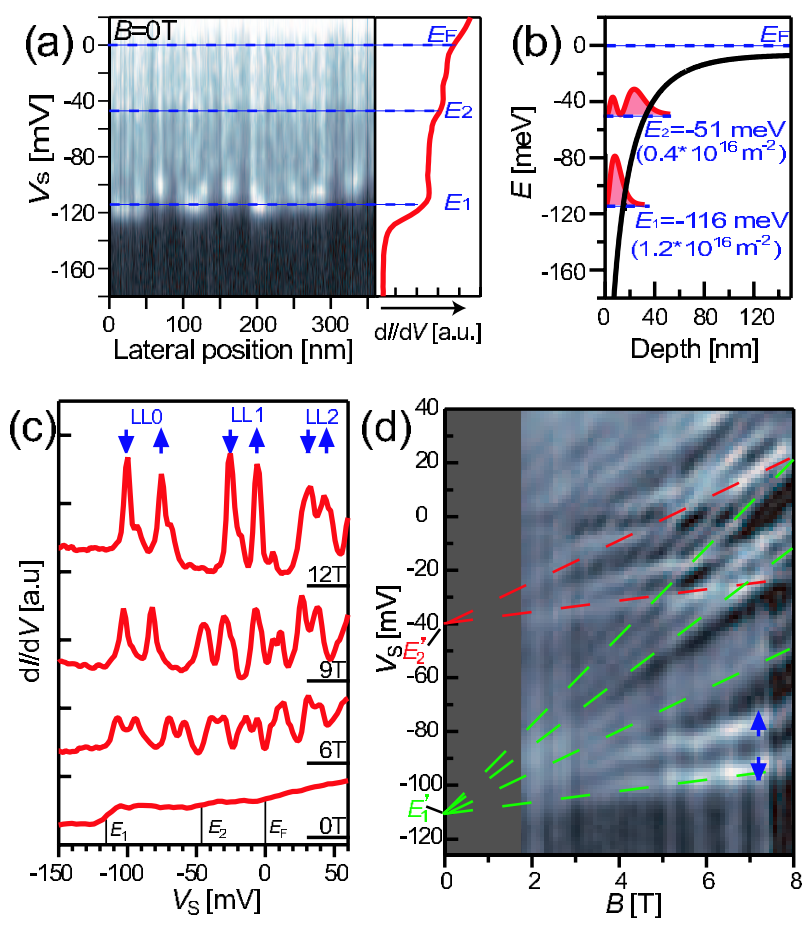

FIG. 1: (a) Color scale plot of $\mathrm{d} I / \mathrm{d} V\left(V_{s}\right)$ measured along a straight line at $B=0 \mathrm{~T}$ (left); corresponding spatially averaged $\mathrm{d} I / \mathrm{d} V\left(V_{s}\right)$ (right); dashed lines mark the Fermi level $E_{F}$ and $i$ th subband energies $E_{i}(i=1,2) ; V_{\text {stab }}=150$ $\mathrm{mV}, I_{\text {stab }}=0.13 \mathrm{nA}, V_{\text {mod }}=2 \mathrm{mV}$. (b) Self-consistently calculated band bending at the InSb surface (black line) and electron-density distribution of $i$ th subband (red areas); $E_{i}$ and electron areal densities $n_{i}$ are marked. (c) $\mathrm{d} I / \mathrm{d} V\left(V_{s}\right)$ at different $B$ recorded at the same lateral position; $E_{i}, E_{F}$, $(d I / d V=0)$-lines on the right, LLs and spin directions are marked; $V_{\text {stab }}=150 \mathrm{mV}, I_{\text {stab }}=0.13 \mathrm{nA}, V_{\text {mod }}=2.0 \mathrm{mV}(0$ $\mathrm{T}), 1.3 \mathrm{mV}(6 \mathrm{~T}), 1.0 \mathrm{mV}(9 \mathrm{~T}), 0.9 \mathrm{mV}(12 \mathrm{~T})$ (d) Experimentally determined Landau fan diagram; green (red) dashed lines: spin-down LLs of the 1st (2nd) subband; arrows: spinup levels of the lowest LL; $V_{\text {stab }}=150 \mathrm{mV}, I_{\text {stab }}=0.10 \mathrm{nA}$, $V_{\text {mod }}=1.5 \mathrm{mV}$.

directly at the LL center [Fig. 2(d)]. This is exactly the expected behavior of an extended drift state at the IQH transition [6, 7]. Fig. 2(i) shows a calculated extended state at $B=12 \mathrm{~T}$ in a 2DES of InSb [23] [24] and the potential disorder provided by the known dopant density of the sample [14]. Good qualitative agreement with the measurement is achieved supporting the interpretation of the coalesced LDOS patterns as extended states. Figure 2(j) shows another extended state recorded on a larger area at different $B$ demonstrating that the coalesced patterns is not restricted to small length scale.

Interestingly, the drift states around the LL center indicate quantum tunneling at the saddle points. Within the classical percolation model, the adjacent drift states are connected at a singular energy at each saddle eventually leading to a localization exponent $\nu=4 / 3$. However, experimental [4], and numerical 7] results for IQH tran- 

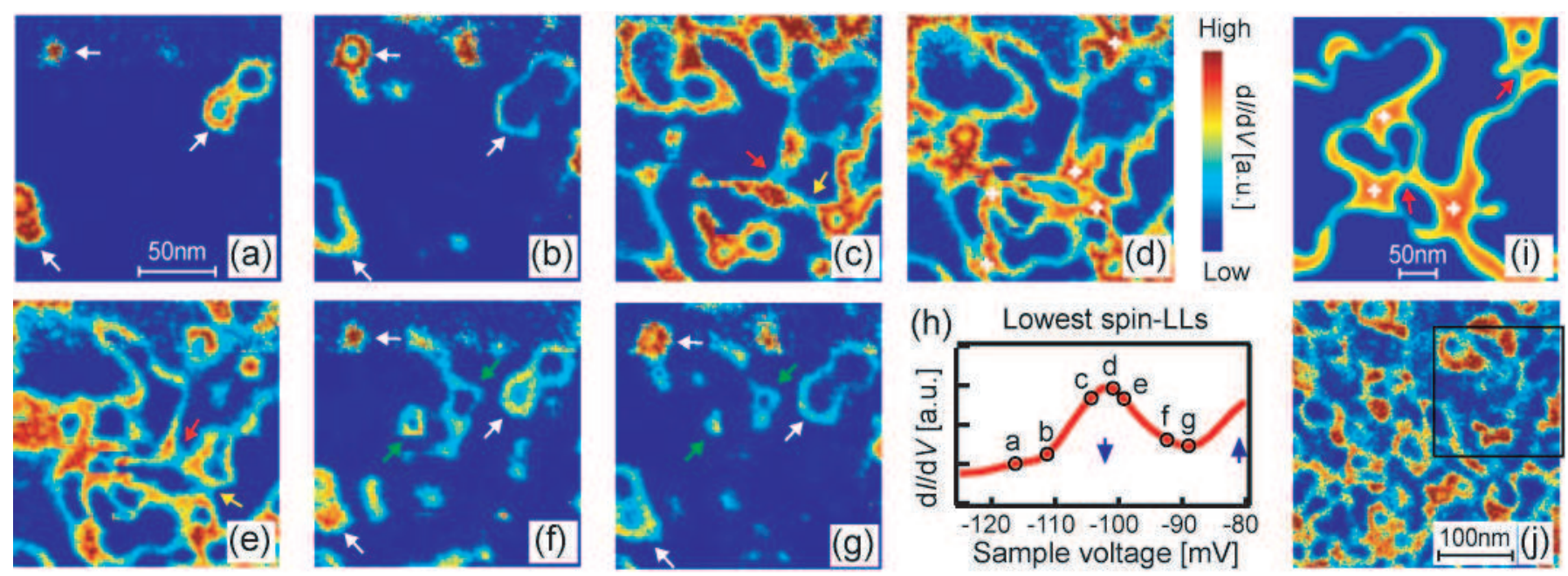

FIG. 2: LDOS of lowest LL. (a)-(g) Measured $\mathrm{d} I / \mathrm{d} V(x, y) ; B=12 \mathrm{~T}, V_{s}=-116.3 \mathrm{mV}$ (a), $-111.2 \mathrm{mV}$ (b), $-104.4 \mathrm{mV}$ (c), $-100.9 \mathrm{mV}(\mathrm{d}),-99.2 \mathrm{mV}(\mathrm{e}),-92.4 \mathrm{mV}(\mathrm{f}),-89.0 \mathrm{mV}(\mathrm{g}), I_{\text {stab }}=0.1 \mathrm{nA}, V_{\text {stab }}=150 \mathrm{mV}, V_{\text {mod }}=1.0 \mathrm{mV} ; \mathrm{same} \mathrm{d} I / \mathrm{d} V$-color scale in each image; white (green) arrows in (a), (b), (f), (g) mark drift states encircling potential minima (maxima); red, yellow arrows in (c), (e) mark tunneling connections existing at identical positions; crosses in (d) mark extended LDOS areas at saddle points. (h) Spatially averaged $\mathrm{d} I / \mathrm{d} V$ curve with circles at the $V_{s}$ used in (a)-(g). (i) Calculated LDOS at the center of $\mathrm{LL} 0 \downarrow$ at $B=12 \mathrm{~T}$; red arrows mark tunneling connections at the saddle points; white crosses mark extended areas. (j) $\mathrm{d} I / \mathrm{d} V$ image close to the center of $\mathrm{LL} 0 \uparrow$ at $B=6 \mathrm{~T}, V_{s}=-99 \mathrm{mV}$; image includes the area of (a)-(g) within the marked rectangle.

sitions revealed $\nu \approx 2.3$. The discrepancy is attributed to quantum tunneling between classically localized drift states [6, 7]. Tunneling connections are indeed visible in our data spreading over an energy range of $5 \mathrm{meV}$. As an example, the red (yellow) arrows in Fig. 2(c) and (e) mark the same connection point at $V_{s}=-104.4 \mathrm{mV}$ and $-99.2 \mathrm{mV}$. LDOS is faintly visible at both positions in both images and sharply rotates by about $90^{\circ}$ between the images. The reason is simply that the tunneling interconnection mediates between valley states at low energies and between hill states at high energies, which are connected via two nearly orthogonal lines. Such weak links are reproduced by the calculation as marked by red arrows in Fig. 2(i) (see also Fig. S3 of [14]). Note that the intrinsic energy resolution of the experiment is $0.1 \mathrm{meV}$ [16], while peaks in the LL fan diagram exhibit a FWHM of $2.5 \mathrm{meV}$ probably due to life-time effects. Thus, broadening due to the energy resolution can hardly account for intensity at the saddles within an energy range of $5 \mathrm{meV}$. Moreover, it cannot explain the change of orientation. Another intriguing observation are the LDOS areas larger than $r_{c}$ around the saddles. They are again visible in experiment [crosses in Fig. 2(d)] and calculation [crosses in Fig. 2(i)] and are probably due to the flat potential at the saddles leading to slow drift speed and, thus, extended LDOS intensity. Notice that both, the spreading of LDOS intensity at the saddles in energy and position, is consistent with previous quantum mechanical calculations [6, 7].

Finally, we discuss the possible influence of the tip. It is known that a mismatch of tip and surface potential leads to band bending within the sample [17, 18]. To avoid this, we used only W-tips exhibiting a minimum of tip-induced band-bending. By analyzing the $d I / d V$ data at $B=0 \mathrm{~T}$ with and without adsorbates, we can safely rule out a work function mismatch between tip and sample larger than $15 \mathrm{meV}$ [14, 17, 18]. The applied $V_{s}$ leads to an additional tip-induced band bending with a lever arm of ten as determined from experiments described in [14]. Thus, we get an additional band bending of less than $12 \mathrm{meV}$. The influence of such small band bendings is tested by the theoretical calculations of a disordered 2DES in $B$-field [14, 23, 24]. The tip-induced potential is added to the disorder potential of the 2DES as a Gaussian with $50 \mathrm{~nm}$ FWHM and amplitude $\left|V_{\text {tip }}\right|<20 \mathrm{meV}$ [17, 18]. The LDOS is calculated at each lateral tip position and its intensity below the tip is displayed as a function of tip position. Fig. 3(a) shows the resulting energy-position plots for three different $V_{\text {tip }}$ at $B=6$ T. Obviously, $V_{\text {tip }}$ leads only to a rigid energetic shift. This result is robust to changes in $B$ and FWHM. Thus, the spatial dependence of the LDOS patterns is not influenced by a small potential mismatch $\left|V_{\text {tip }}\right|<20 \mathrm{meV}$.

To substantiate this result, Figs. 3(b)-(e) show experimentally determined voltage-position plots in comparison with calculated energy-position plots [14, 23] at $B$ $=6$ and 12 T. Spin-split LLs fluctuating in energy are visible as meandering pairs of lines. The theoretical calculations do not require free parameters by neglecting the tip-induced potential. They reproduce the experimental trends of spatially fluctuating spin resolved LLs. The fluctuation length is $80 \%$ larger and the fluctuation 

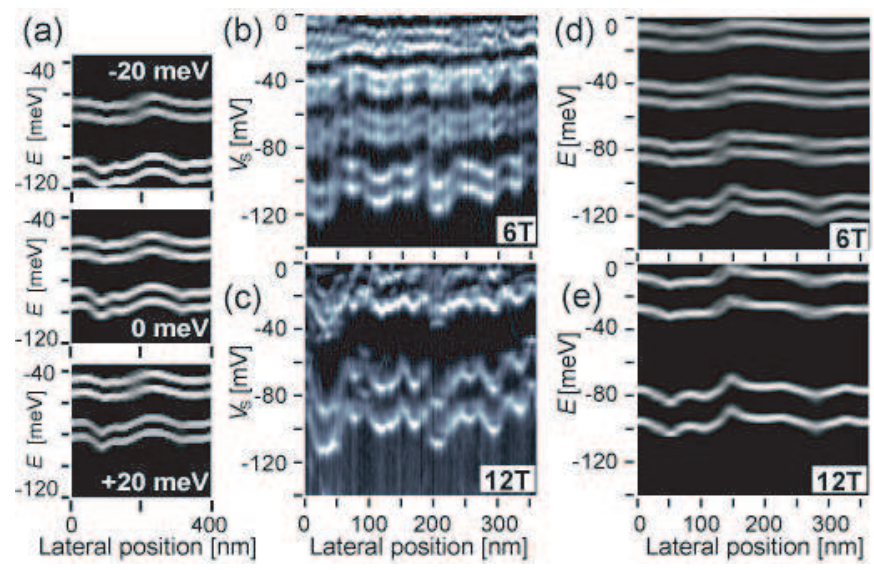

FIG. 3: (a) Calculated $\operatorname{LDOS}(E, x)$ at $B=6 \mathrm{~T} ; V_{\text {tip }}=0$ and $\pm 20 \mathrm{meV}$ as marked; $m^{*} / m_{e}:=0.02,|g|:=28$ [23] (b), (c) Measured $\mathrm{d} I / \mathrm{d} V\left(V_{s}, x\right)$ at $B=6$ and $12 \mathrm{~T} ; x$ : lateral position along straight line; $I_{\text {stab }}=0.13 \mathrm{nA}, V_{\text {stab }}=150 \mathrm{mV}, V_{\text {mod }}=$ $1.3 \mathrm{mV}$ (b), $0.9 \mathrm{mV}$ (c). (d), (e) Calculated $\operatorname{LDOS}(E, x)$ at $B=6$ and $12 \mathrm{~T} ; V_{\mathrm{tip}}=0 \mathrm{meV}$.

amplitude $40 \%$ lower than in the experiment. This is most likely due to the neglected inhomogeneous charge distribution of the adsorbates, which would increase the potential disorder given by the bulk dopants. Indeed, as shown in Fig. S1 of [14], the spatial distribution of adsorbates is related to the resulting drift states, and the correspondence is improved by adding surface charges to the calculation (not shown). However, since many details of the adsorbate potential are not known, we omit its inclusion, thereby avoiding the use of further parameters. Note that the parameter-free calculation and the experiment quantitatively exhibit the same trends with $B$-field and energy, i.e. a $30 \%$ increase of fluctuation amplitude between 6 and $12 \mathrm{~T}$ and a $60 \%$ decrease of fluctuation amplitude between LL0 and LL3 at $B=6$ T. Both effects are explained straightforwardly by the fact that drift states can only probe potential fluctuations down to length scales of $r_{c}=\sqrt{(2 n+1) \cdot \hbar /(|e| B)}$ ( $n$ : LL index).

In summary, our STS experiments provide the first direct observation of wave functions across an IQH transition. They reveal the development from localized to extended states including indications of the theoretically predicted quantum tunneling at saddle points of the disorder potential. We probe states well away from the Fermi level, and thus artificially exclude the influence of electron-electron interaction, thereby considering a theoretically pure IQH transition, which is regarded as a hallmark in the theoretical description [6, 7, 8, [9]. Indeed, single-particle calculations can largely reproduce our experimental results. In principle, the experiments can be extended to 2DES states at $E_{F}$ by using p-type samples. Thus, we could add the electron-electron interaction, which reveals a wealth of further quantum phase transitions, in future experiments [9, 11]. However, already the present results go far beyond previous results 12, 13] by providing the states at the quantum phase transition, a LL fan diagram and a detailed theoretical reproduction of the observed experimental LDOS features.

We thank F. Evers, H. Akera, G. Bauer, M. B. Santos, D. Haude, F. Marczinowski, S. v. Oehsen, and G. Meier for helpful discussions and the DFG program "QuantumHall systems" as well as SFB 508-B4 for financial support. Part of the calculations were performed at ISIC, Iwate University and Cyberscience Center, Tohoku University as well as at the UK National Grid Service.

* Electronic address: hashi@mail.tains.tohoku.ac.jp

† Present address: Department of Physics, Cornell University, Ithaca NY 14853, USA

[1] S. Sachdev in Quantum Phase Transitions, (Cambridge Univ. Press, Cambridge, 1999).

[2] E. L. Shangina and V. T. Dolgopolov, Phys. Usp. 46, 777 (2003).

[3] Y. Dubi et al., Phys. Rev. Lett. 94, 156406 (2005).

[4] S. Koch et al., Phys. Rev. Lett. 67, 883 (1991); F. Hohls et al., Phys. Rev. Lett. 88, 036802 (2002).

[5] R. Joynt and R. E. Prange, Phys. Rev. B 29, 3303 (1984).

[6] T. Ando, J. Phys. Soc. Jpn. 53, 3101 (1984).

[7] B. Kramer et al., Phys. Rep. 417, 211 (2005).

[8] A. D. Mirlin et al, Ann. D. Phys. 5, 281 (1996).

[9] F. Evers and A. Mirlin, arXiv 0707.4378v1 (2007).

[10] M. Morgenstern in Scanning Probe Microscopy, ed. by S. Kalinin and A. Gruwerman, (Springer, New York, 2007); S. H. Tessmer et al., Nature 392, 51 (1998); A. Baumgartner et al., Phys. Rev. B 76, 085316 (2007); E. Ahlswede et al., Physica B 298, 562 (2001).

[11] S. Ilani, et al., Nature 427, 328 (2004).

[12] M. Morgenstern et al., Phys. Rev. Lett. 90, 056804 (2003).

[13] Y. Niimi et al., Phys. Rev. Lett. 97, 236804 (2006).

[14] See EPAPS Document No. for supplementary texts, figures and movie. For more information on EPAPS, see http://www.aip.org/pubservs/epaps.html

[15] The Cs induces downwards band bending within the InSb and, thereby, creates the 2DES. See M. G. Betti et al., Phys. Rev. B 63, 155315 (2001).

[16] J. Wiebe et al., Rev. Sci. Instrum. 75, 4871 (2004).

[17] R. Dombrowski et al., Phys. Rev. B 59, 8043 (1999).

[18] M. Morgenstern et al., Phys. Rev. Lett. 89, 136806 (2002).

[19] S. Abe et al., Phys. Rev. B 66, 205309 (2002).

[20] R. Masutomi et al., Appl. Phys. Lett. 90, 202104 (2007).

[21] U. Merkt et al., Phys. Rev. B 34, 7234 (1986);

[22] A.N. Chantis et al., Phys. Rev. Lett. 96, 086405 (2006).

[23] We use constant values $m^{*} / m_{e}=0.02,|g|=28$ determined by energetically averaging the Landau and spin level separation in Fig. 3(b).

[24] The results do not change when including many-particle interactions via Hartree-Fock, see C. Sohrmann and R.A. Römer, New J. Phys. 9, 97 (2007). 